\title{
OTOSCOPIC EXAMINATION
}

Berkay Kef ${ }^{1}$, Nur Gülce İșkan², Kemal Kef ${ }^{3}$

1 Okan University School of Medicine, Istanbul, TURKEY

2 Trakya University School of Medicine, Edirne, TURKEY

3 Department of Otolaryngology, Private Kesan Hospital, Edirne, TURKEY

Abstract: Ear related diseases are common in both otolaryngology clinics and family practice since all age groups can get affected. Due to the frequency and the range of diseases of the ear, it is very important to carry out a thorough examination and make a correct diagnosis. Otoscopes are very useful tools used for otoscopic examination. Although head mirrors and head lambs can be used for the examination of the ear, otoscopes provide a better field of vision and a more detailed sight. There are two main types of otoscopes; portable and wall-mounted. However, the examination should not be limited to the outer ear. It is the examiner's responsibility to check the area around the ear for abnormalities. Otoscopic examination is a convenient method for making a correct diagnosis. Therefore, every medical doctor should be able to perform a proper otoscopic examination and make a correct diagnosis.

Keywords: Ear, otitis, otoscopes, tympanic membrane

\section{INTRODUCTION}

Diseases regarding the ear are common in not only otolaryngology clinics but also in family practice. It is an important disease group that alarms patients and their families (especially in pediatric age groups) to consult an emergency service for treatment. Detailly taken medical history of the patient is important as in all diseases (1).

Ear: Consists of 3 anatomical parts; Outer Ear: Auricle (pinna) and the ear canal, and Middle Ear: Tympanic membrane, the ossicles, tympanic cavity, mastoid cavity and the Eustachian tube and Inner Ear: Cochlea and vestibular structures.

Otoscopic examination is essential for examining the ear canal and the eardrum with a light source. Examination of the eardrum can reveal information about some of the middle ear pathologies (2). Many tools and light sources are used for this purpose. Otoscopic examination can also be performed by reflecting the light from the light source into the ear canal through an ear spectrum via a head mirror. For this examination chair for the patient's head to rest on, a head mirror, various sizes of spectrums and a light source are needed. This method provides a better sense of depth due to its binocular view. However, carrying the equipment for the inpatients, examining non-cooperative patients and children can be difficult. This method does not provide any magnification; ear canal and the eardrum are seen in their actual size. Directing the light with the head mirror also takes practice.

Otoscope: Today, otolaryngologists and family practitioners prefer using an otoscope. Otoscopes are light, portable tools that include a light source and a lens system, providing magnification. Fiber optic lights or small light bulbs are used as the light source. There are many types of portable otoscopes as well as wall mounted otoscopes in the clinics (Figure 1A, B, C). Advances in the field of medical engineering provide more variety and lower prices.
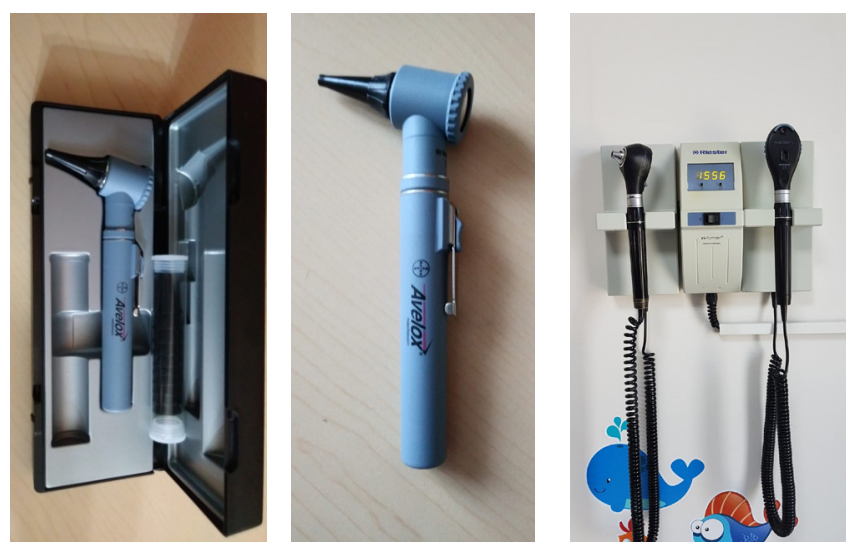

Figure 1: A: Portable otoscope in its case with various sizes of spare speculums, B: Portable otoscope, C: Wall mounted otoscopes. 
Otoscopes generate brighter light (compared to head mirrors) and magnify the image. They can be carried easily for inpatient examination. However, interventions of the outer ear and the eardrum are harder. There is no sense of depth due to monocular vision.

Otolaryngology microscopes and endoscopes of proper size can also be used to examine the ear. Microscopic and endoscopic examination provide a clearer, magnified view. For troubled cases where a surgery is considered it is a must to use microscopes and endoscopes. Otoscopes are preferred in clinical use due to their convenience.

Auricle: Pinna (external ear) comes in all shape and sizes and changes from person to person. It has an elastic cartilaginous structure. The overlying skin is firmly attached to the cartilage. Only the earlobe has fatty tissue and skin instead of cartilage. Prominent ear occurs in the absence of antihelix or when the angle between the head and the ear is greater than $30^{\circ}$. There are no physiological disadvantages of prominent ear (3). Auricle must also be checked before examining the ear canal. Congenital anomalies, symmetry, size, hypoplasia or atresia, the presence of hematoma must be evaluated. Discoloration of the skin, tophus, skin eruption, infection, tumor lesions, scar tissue, chondrite and perichondritis allergic reaction symptoms, periauricular fistulas and vascular pathologies must be taken into consideration (4-7). Pressure should be applied to tragus to check for pain (pain is present in; otitis externa in adults and acute otitis in children). The pain and the clicking sound when pressure is applied to the front end of tragus are signs of temporomandibular joint disorders (Costen Syndrome) (8). The backside of the auricle and the upper surface of mastoid must be evaluated. Mastoid surface and the nearby area must be checked for lymphadenopathy, edema, swelling, rash, depression (caused by mastoidectomy), scar tissue, trauma and signs of tumor (Figure 2, 3, 4).

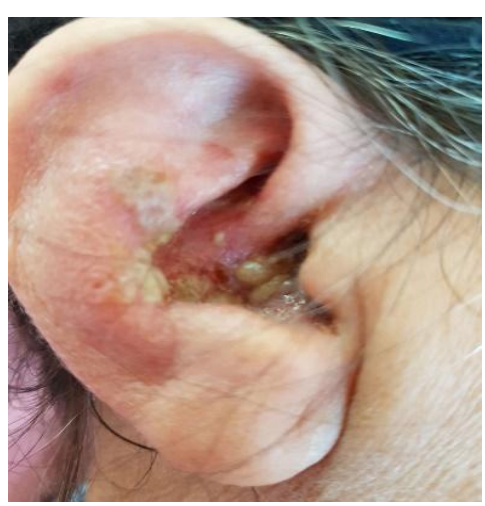

Figure 2: Herpetic eruption (Herpes zoster oticus).

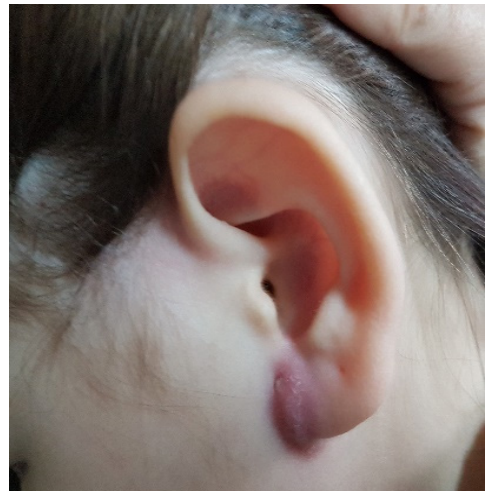

Figure 3: Fistula on the front side of the ear.

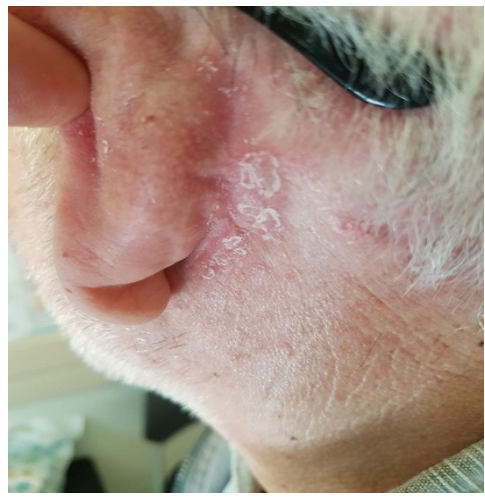

Figure 4: Psoriasis lesions on the backside of the ear.

Ear Canal: There are two structures present; outer cartilaginous canal and inner bony canal. The canal is not straight. It has an "S" shape. The length of the back wall is $25 \mathrm{~mm}$ and the front wall is $30 \mathrm{~mm}$ in adults. Hence the eardrum is placed at an angle from back to the front. One-third of the ear canal is composed of the cartilaginous canal, the overlying skin and cutaneous adnexa. There are two fissures on the front wall of the cartilaginous canal (Santorini fissures). The cartilage is not enclosed postero-superiorly (incisura terminalis). The skin on the cartilaginous region is thick. It contains ceruminous glands that are not present anywhere else in the body. Sebaceous glands and hair follicles are also present in this region. Skin is very thin in the bony canal. Since it is firmly attached to the bone, careless and harsh interventions cause bleeding and severe pain (2). There are no cutaneous adnexa in the bony canal. The radius of the canal is 7 to $9 \mathrm{~mm}$. Vertical radius is larger. Innervation of the ear canal is provided by the 5 th, 8 th, 10th cranial nerves and 3rd cervical nerve. Branch of Vagus in this region is called the Arnold nerve (4). It might cause a fit of coughing and bradycardia while operating on the ear canal. Intense coughing might occur especially in elder patients preventing the perfor- 
ming of the operation. The ear canal must be examined without using a speculum or an otoscope at first. Bleeding at the canal, mucoid or purulent discharges must be evaluated (9). Serous discharges following a head trauma might be a sign for cerebrospinal fluid leakage. Temporal fractures must come to mind in the presence of bloody discharge. Middle and external ear tumors and bullous hemorrhage otitis, foreign bodies inside the ear must be considered if trauma is not present in the patient's history (10). Furuncle, osteoma, otitis externa with severe pain, foreign bodies, cerumen accumulation and rigid plugs can be seen early on (Figure 5) (11).

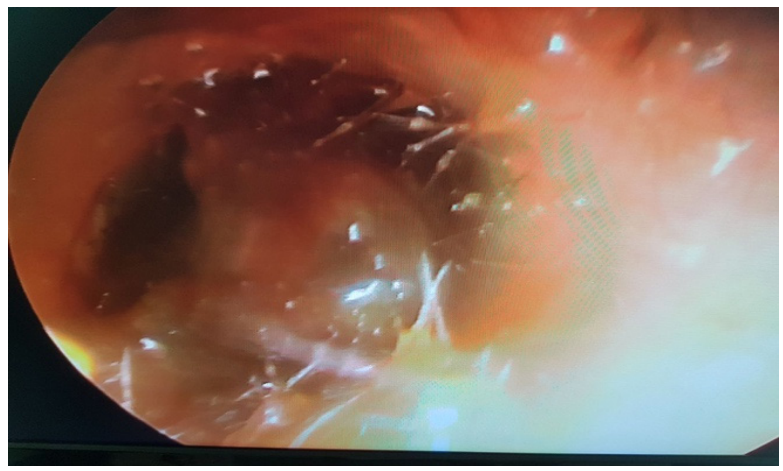

Figure 5: Earwax blockage.

\section{Otoscopic Examination}

During otoscopic examination, the patient should be sitting on the examination chair and his head should be stable. The patient should be asked to turn his head left while the practitioner is examining the right ear and turn right while examining the left ear. Children should be examined while they are seated on one of their parent's lap and their heads should be held on the chest of the parent and turned to other side. The speculum which is used in ear canal should not be oversized. However, it should be broad enough to provide a good image and to insert an aspirator or other tools in necessary conditions. The auricle should be pulled backwards and upwards in adults because of the curve in the ear canal. In children, there are two methods: pulling the auricle backwards and downwards while the entrance of ear canal is observed, or it should be pulled backwards. The otoscope should be led through the ear canal. The speculum which is at the end of the otoscope may cause severe pain or bleeding if it is pushed quickly. Therefore, the movements of the practitioner should be smooth and controlled. Otoscope should be held as close to the front end as possible, similar to holding a pencil. The otoscope should be led looking into the ear. The examination of babies and children are more difficult than adults' examination. The practitioner should be slow and careful so as not to frighten the children during the examination. It is difficult to properly examine a crying and anxious child. Especially in the examinations in primary care units, the color change - rash due to crying in tympanic membrane may lead to misdiagnosis (12).

Ear canal (EA): Obstructions in the ear canal, stenosis, pushed-in earwax, foreign bodies, localized or diffuse external otitis, furuncle formation, otomycosis, fracture line due to trauma, serous, purulent, hemorrhagic discharge, exostosis or osteoma, tumoral changes or tumors should be examined (13-15). Posterosuperior meatal wall should be examined whether for any signs of depression. Earwax or foreign bodies which are blocking the ear canal should be removed properly. Living foreign bodies such as insects or flies in the ear canal should be killed by applying eardrops and then, aspirated or taken out with an alligator forceps (16). In severe external otitis that causes narrowness, tympanic membrane may be difficult to examine. If the otoscope is forced forwards in order to see the tympanic membrane, severe pain or bleeding might occur. Initially, external otitis should be treated (Figure 6-9) $(13,17,18)$.

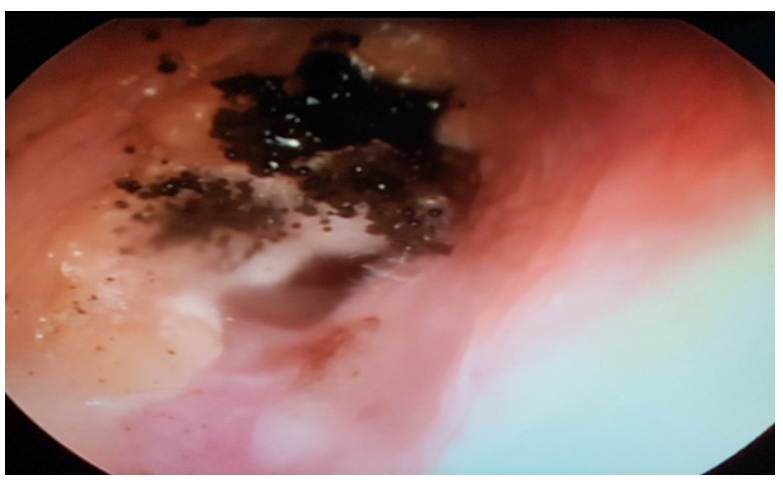

Figure 6: Otomycosis in the external auditory canal.

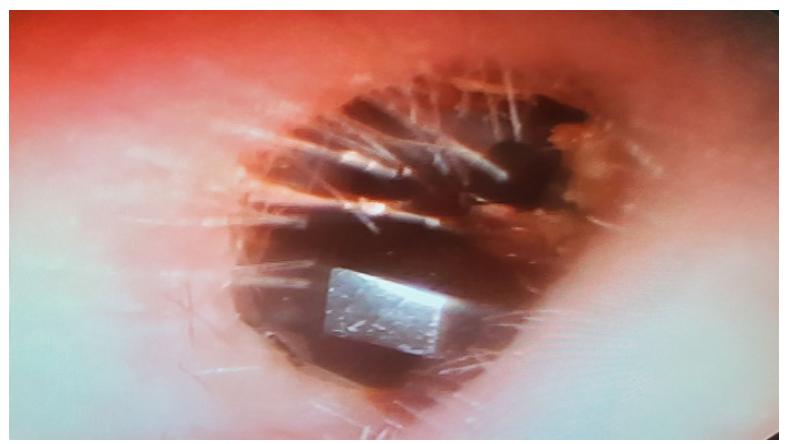

Figure 7: Foreign body. 


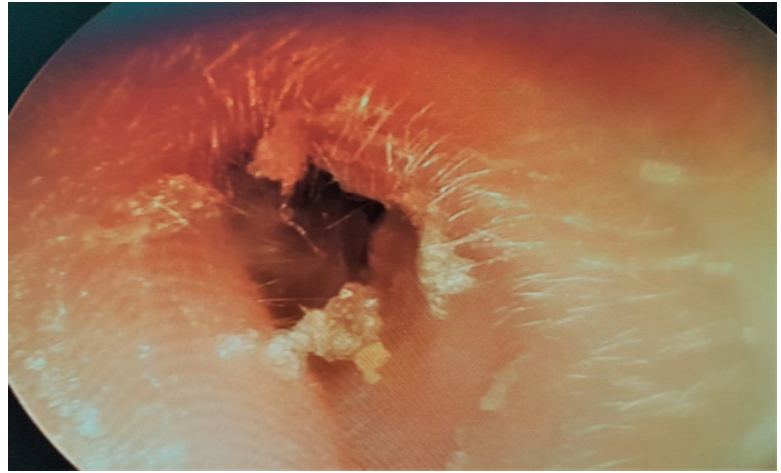

Figure 8: Seborrheic dermatitis.
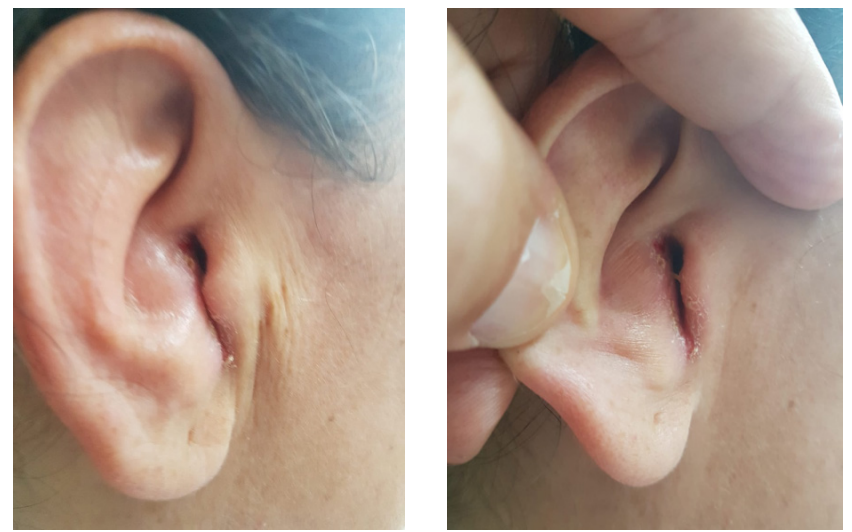

Figure 9: A, B: Extreme narrowness of the ear canal due to an untreated infection.

Eardrum: It is placed at the end of the ear canal and the border of middle ear. It is iridescent, transparent and oval. It has an oblique placement according to the ear canal. It is sloping from top to bottom and front to back. Its vertical diameter is $9-10 \mathrm{~mm}$, horizontal diameter is $8-9 \mathrm{~mm}$. The surface which is facing the canal is covered with epithelium. This surface is considered to be included in the outer ear. The surface which is facing the middle ear is covered with endothelium. In the middle of these surfaces, there is a fibrous layer. It has two different parts: Pars tensa and Pars flaccida. The fibrous layer is very small in pars flaccida (Shrapnell's membrane) (3). Therefore, it is neglected. In addition, there is no fibrous ligament in this layer. The different structure of pars flaccida is important in the formation of retraction pockets and cholesteatomas. It is defined in 4 quadrants as upper front, lower front, upper back and lower back according to the vertical line passing through the long arm of malleus and the horizontal imaginary line passing perpendicular to this line. The tip of the eardrum is conical towards the middle ear. The most depressed part is umbo which is formed by the tip of malleus. It reflects the light because of its structure. This triangle of light is called the Politzer's triangle whose apex is in umbo and base is towards the front to the annulus. This shows that the structure of the eardrum and its placement angle in the ear canal is normal $(3,4)$.

The otoscopic evaluation of eardrum: All of the quadrants mentioned above and the general structure of the eardrum should be evaluated. Pneumatic otoscope or Siegel's speculum can be used for evaluating its mobility (19). Rash due to infection, hemotympanum, discharge, perforation, retraction pockets, the posture of malleus, umbo, the presence of Politzer's triangle are evaluated. Air-fluid levels behind the earlobe, presence of red-blue reflection in glomus tympanicum and glomus jugulare, the long arm of incus should be examined to the extent that the transparent structure of the membrane permits. Perforations should be evaluated for central or marginal formation. Marginal perforations have a risk for cholesteatoma formation. In congenital cholesteatomas, a white-colored mass image may be seen. If there is no active infection, the Eustachian tube can be examined by Valsalva maneuver in otoscopic examination. In newly formed traumatic perforations, blood on the perforation edges and tympanic membrane leaves which are curled backwards may be seen. Calcifications, thickenings, thinned areas due to the infections in tympanic membrane might be seen. The presence of Brown's sign or Schwartz sign should be evaluated (9). If there is a broad perforation, mucosa of the middle ear, promontorium, fenestrae and ossicles may be examined (Figure 10-14).

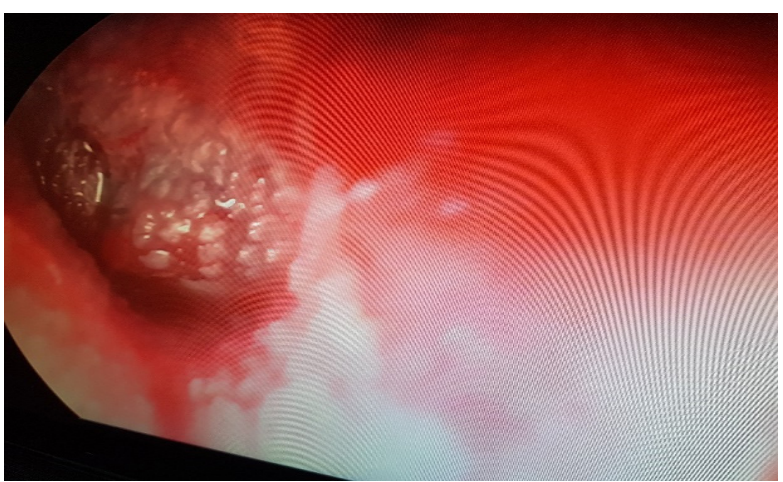

Figure 10: Bullous myringitis. 


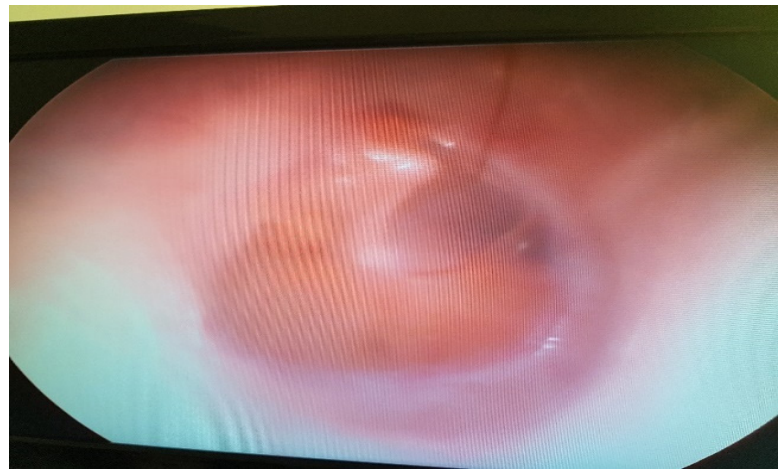

Figure 11: Acute otitis.

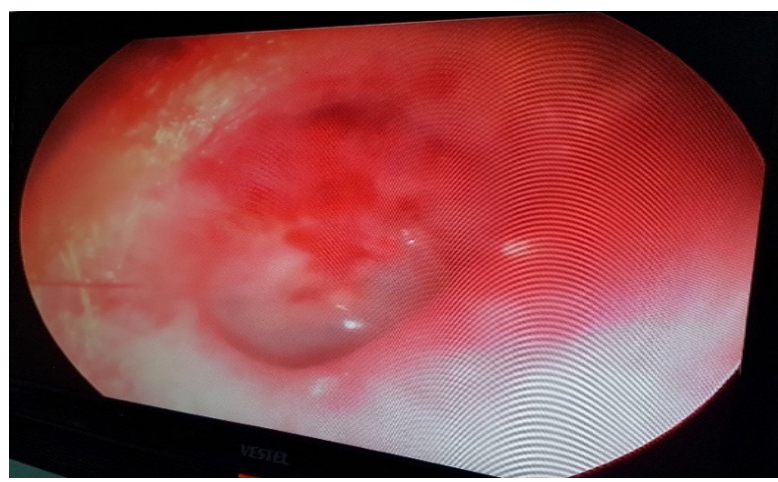

Figure 12: Serous otitis.

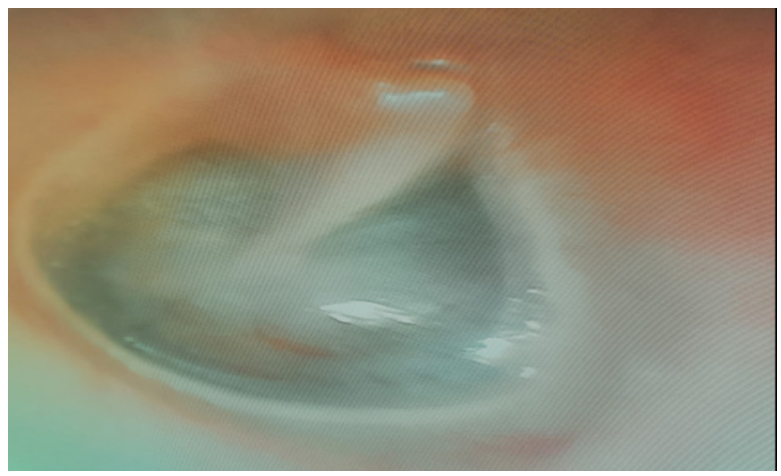

Figure 13: Serous otitis, "blue" tympanic membrane due to delayed treatment.

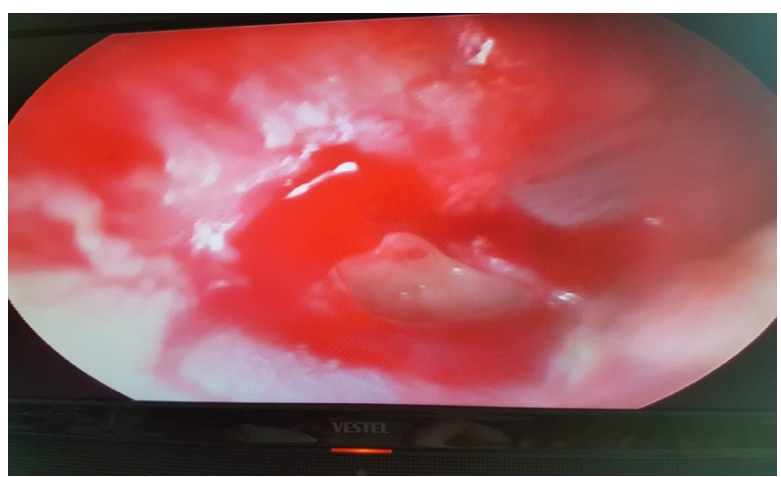

\section{CONCLUSION}

Since ear related diseases are very common and patients from all age groups can get affected, it is necessary to carry out a proper examination and make a correct diagnosis. Otoscopes provide a good field of vision for the examination of the ear. Therefore, every medical doctor should be able to perform a proper otoscopic examination in order to make a correct diagnosis.

\section{REFERENCES}

1. Austin DF. Diseases of the external ear. In: Ballenger JJ, editor. Diseases of the Nose, Throat, Ear, Head And Neck. Philadelphia: Lea \& Febiger; 1991.p.106980.

2. Selesnick SH. Diseases of the external auditory canal. Otolarygol Clin North Am 1996;8:725-886.

3. Asım Aslan. Kulak Anatomisi. In: Can Koç, editor. Kulak Burun Boğaz Hastalıkları ve Baş Boyun Cerrahisi. Ankara: Güneş Kitabevi; 2004.p.45-61.

4. External auditory canal. In: Benjamin B, Bingham B, Hawke M, editors. A Colour Atlas of Otorhinolaryngology. London and Philadelphia: Martin Dunitz and JB Lippincott; 1995.p.36-65.

5. Akyıldız AN. Kulak hastalıkları ve mikrocerrahisi. Ankara: Bilimsel Tip Yayınevi;1998.

6. Hawke M, Bingham B, Stammberger $\mathrm{H}$ et al, editors. Otolaringoloji Tanı El Kitabı. London:Martin Dunitz;2012.

7. Dermatological importance of the ear region. In: Baykal C, Yazganoğlu KD, editors. Dermatological Diseases of the Nose and Ears: an Illustrated Guide. Berlin: Springer; 2016.p.63-117.

8. Găvoronskiĭ IV, Iordanishvili AK, Voitiatskaia IV et al. The peculiarities of petrotympanic fissure topography in costen sydrome and possible causes of its development. Morfologia 2014;145(2):58-62.

9. Abdelazeem M, Gamea A, Mubarak H et al. Epidemiology, causative agents, and risk factors affecting human otomycosis infections. Turk J Med Sci 2015;45(4):820-6.

Figure 14: Central traumatic perforation. 
10. Wright T. Middle-ear pain and trauma during air travel. BMJ Clin Evid 2015.

11. Clark GW, Pope SM, Jaboori KA. Diagnosis and treatment of seborrheic dermatitis. Am Fam Physician 2015;91(3):185-90.

12. Guss J, Ruckenstein MJ. Infections of the external ear. In: Flint PW, Haughey BH, Lund VJ et al, editors. Cummings Otolaryngology: Head and Neck Surgery.

New York: Elsevier/Saunders; 2015.p.1944-62.

13. Joy SD. Otoscopic findings accurately diagnose otitis media. American Journal of Nursing 2011;111(6):14-5.

14. Kuo CY, Lin YY, Wang CH. Painful rash of the auricle: herpes zoster oticus. Er Nose Throat 2014;93(12):47.

15. Maqbool M. Textbook of Ear, Nose and Throat Diseases. 11th edition. New Delhi: J.P.Medical; 2013.

16. DiMuzio JJ, Deschler DG. Emergency department management of foreign bodies of the external ear canal in children. Otol Neurotol 2002;23:473-5.

17. Monasta L, Ronfani L, Marchetti F et al. Burden of disease caused by otitis media: systematic review and global estimates. PLoS ONE 2012;7(4):e36226.

18. Qureishi A, Lee Y, Belfield K et al. Update on otitis media - prevention and treatment. Infect Drug Resist 2014;7:15-24.

19. Koçyiğit M, Uzun C. Comparison of the diagnostic accuracies of four main otoscopic examination methods. International Journal of Surgery and Medicine 2017;3(2):78-84. 